\title{
Identification of candidate genes and prognostic value analysis in patients with PDL1-positive and PDL1-negative lung adenocarcinoma
}

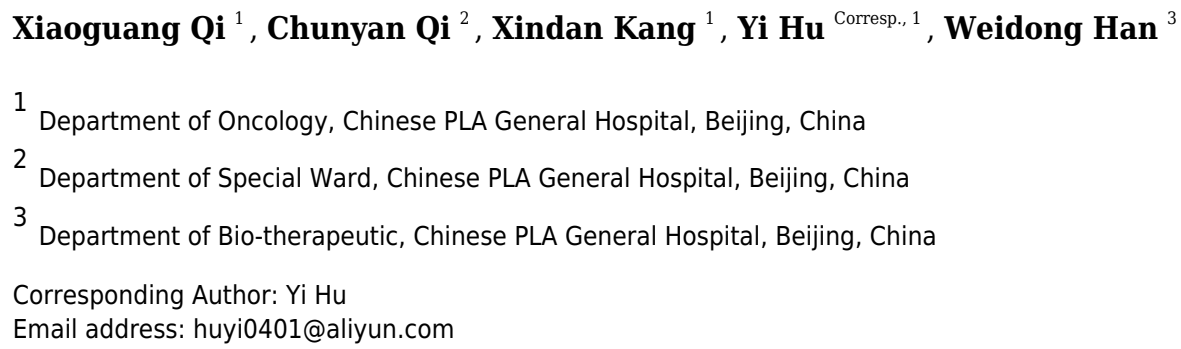

Background: Increasing bodies of evidence reveal that targeting a programmed cell death protein 1 (PD-1) monoclonal antibody is a promising immunotherapy for lung adenocarcinoma. Although PD receptor ligand 1 (PDL1) expression is widely recognized as the most powerful predictive biomarker for anti-PD-1 therapy, its regulatory mechanisms in lung adenocarcinoma remain unclear. Therefore, we conducted this study to explore differentially expressed genes (DEGs) and elucidate the regulatory mechanism of PDL1 in lung adenocarcinoma. Methods: The GSE99995 data set was obtained from the Gene Expression Omnibus (GEO) database. Patients with and without PDL1 expression were divided into PDL1-positive and PDL1-negative groups, respectively. DEGs were screened using R. The Gene Ontology (GO) database and Kyoto Encyclopedia of Genes and Genomes (KEGG) were analyzed using the Database for Annotation, Visualization and Integrated Discovery . Protein-protein interaction (PPI) networks of DEGs was visualized using Cytoscape, and the MNC algorithm was applied to screen hub genes. A survival analysis involving Gene Expression Profiling Interactive Analysis was used to verify the GEO results. Mutation characteristics of the hub genes were further analyzed in a combined study of five datasets in The Cancer Genome Atlas (TCGA) database. Results: In total, 869 DEGs were identified, 387 in the PDL1-positive group and 482 in the PDL1negative group. GO and KEGG analysis results of the PDL1-positive group mainly exhibited enrichment of biological processes and pathways related to cell adhesion and peroxisome proliferators-activated receptors (PPAR) signaling pathway, whereas biological process and pathways associated with cell division and repair were mainly enriched in the PDL1negative group. The top 10 hub genes were screened during the PPI network analysis. Notably, survival analysis revealed BRCA1, mainly involved in cell cycle and DNA damage responses, to be a novel prognostic indicator in lung adenocarcinoma. Moreover, the 
prognosis of patients with different forms of lung adenocarcinoma was associated with differences in mutations and pathways in potential hub genes. Conclusions: PDL1positive lung adenocarcinoma and PDL1-negative lung adenocarcinoma might be different subtypes of lung adenocarcinoma. The hub genes might play an important role in PDL1 regulatory pathways. Further studies on hub genes are warranted to reveal new mechanisms underlying the regulation of PDL1 expression. These results are crucial for understanding and applying precision immunotherapy for lung adenocarcinoma. 
1 Identification of candidate genes and prognostic value analysis in patients with PDL1-

2 positive and PDL1-negative lung adenocarcinoma

3 Xiaoguang $\mathrm{Qi}^{1}$, Chunyan $\mathrm{Qi}^{2}$, Xindan Kang ${ }^{1}, \mathrm{Yi} \mathrm{Hu}{ }^{1 *}$ and Weidong $\mathrm{Han}^{3}$

$4{ }^{1}$ Department of Oncology, Chinese PLA General Hospital, Beijing, China

$5 \quad 2$ Department of Special Ward, Chinese PLA General Hospital, Beijing, China

$6{ }^{3}$ Department of Bio-therapeutic, Chinese PLA General Hospital, Beijing, China

7

8

9 Corresponding Author: $\mathrm{Yi} \mathrm{Hu}$,

10 No. 28 Fuxing Road, Haidian District, Beijing, 100853, China.

11 Email address: huyi0401@aliyun.com. 
ABSTRACT

Background: Increasing bodies of evidence reveal that targeting a programmed cell death protein 1 (PD-1) monoclonal antibody is a promising immunotherapy for lung adenocarcinoma.

Although PD receptor ligand 1 (PDL1) expression is widely recognized as the most powerful predictive biomarker for anti-PD-1 therapy, its regulatory mechanisms in lung adenocarcinoma remain unclear. Therefore, we conducted this study to explore differentially expressed genes (DEGs) and elucidate the regulatory mechanism of PDL1 in lung adenocarcinoma. Methods: The GSE99995 data set was obtained from the Gene Expression Omnibus (GEO) database. Patients with and without PDL1 expression were divided into PDL1-positive and PDL1-negative groups, respectively. DEGs were screened using R. The Gene Ontology (GO) database and Kyoto Encyclopedia of Genes and Genomes (KEGG) were analyzed using the Database for Annotation, Visualization and Integrated Discovery. Protein-protein interaction (PPI) networks of DEGs was visualized using Cytoscape, and the MNC algorithm was applied to screen hub genes. A survival analysis involving Gene Expression Profiling Interactive Analysis was used to verify the GEO results. Mutation characteristics of the hub genes were further analyzed in a combined study of five datasets in The Cancer Genome Atlas (TCGA) database. Results: In total, 
38869 DEGs were identified, 387 in the PDL1-positive group and 482 in the PDL1-negative group.

39 GO and KEGG analysis results of the PDL1-positive group mainly exhibited enrichment of

40 biological processes and pathways related to cell adhesion and peroxisome proliferators-

41 activated receptors (PPAR) signaling pathway, whereas biological process and pathways

42 associated with cell division and repair were mainly enriched in the PDL1-negative group. The

43 top 10 hub genes were screened during the PPI network analysis. Notably, survival analysis

44 revealed $B R C A 1$, mainly involved in cell cycle and DNA damage responses, to be a novel

45 prognostic indicator in lung adenocarcinoma. Moreover, the prognosis of patients with different

46 forms of lung adenocarcinoma was associated with differences in mutations and pathways in

47 potential hub genes. Conclusions: PDL1-positive lung adenocarcinoma and PDL1-negative lung

48 adenocarcinoma might be different subtypes of lung adenocarcinoma. The hub genes might play

49 an important role in PDL1 regulatory pathways. Further studies on hub genes are warranted to

50 reveal new mechanisms underlying the regulation of PDL1 expression. These results are crucial

51 for understanding and applying precision immunotherapy for lung adenocarcinoma.

\section{INTRODUCTION}

53 Non-small-cell lung cancer (NSCLC) is a leading malignancy threatening human life and health

54 worldwide (Herbst, Morgensztern, \& Boshoff,2018; Zhang et al.,2019). Lung adenocarcinoma is

55 the most common type of NSCLC, and it is a more heterogeneous subtype of NSCLC compared

56 with squamous cell carcinoma. Therefore, its individualized treatment and prognosis have

57 attracted considerable attention; understanding its biological characteristics is necessary for

58 achieving such individualized treatment and prognosis. In the past decade, studies have identified 
59

60

61

62

63

tyrosine kinase inhibitors (TKIs) targeting epidermal growth factor receptor (EGFR), anaplastic lymphoma kinase (ALK), and ROS proto-oncogene 1 (ROS1) as potential therapies for lung adenocarcinoma on the basis of genotyping (Sgambato et al., 2018; Singhi et al., 2019).

Molecular targeted therapy based on these sensitive targets has considerably enhanced overall survival (OS) in lung adenocarcinoma. Despite the progress in the treatment of lung adenocarcinoma, the mortality rate among patients with advanced lung adenocarcinoma remains high, with the 5-year survival rate being approximately $15 \%$ in patients with advanced NSCLC (Blandin Knight et al., 2017). Nevertheless, a breakthrough was achieved in terms of immunotherapy for cancer according to recent reports, with immune checkpoint inhibitors prolonging survival in some patients with various cancer types (Yu \& Wang, 2018; Lorigan \& Eggermont, 2019; Morse, Hochster, \& Benson, 2020). In particular, targeting programmed cell death protein 1 (PD-1) monoclonal antibodies is rapidly becoming a promising therapeutic approach for NSCLC treatment (El-Osta \& Jafri, 2019). Patients with $>50 \%$ PDL1 expression treated using anti-PD-1 therapy showed a longer progression-free survival (PFS) period than did those treated using standard chemotherapy as the first-line treatment for NSCLC. Furthermore, in the past few years, some of these antibodies have been successfully commercialized and approved as first- and second-line treatments for advanced NSCLC (Gridelli et al., 2018; Dafni et al., 2019). In particular, immunotherapy is different from traditional chemotherapy or targeted therapy in that it has durable clinical benefits and fewer side effects in general (Suresh et al., 2018). Hence, immunotherapy is a promising therapy for lung adenocarcinoma; however, its application is limited to a subtype of patients with lung adenocarcinoma. Individualized 
80

81

82

83

84

85

immunotherapy guided by predictive biomarkers is crucial for improving the prognosis of lung adenocarcinoma.

Recent studies have proposed PDL1 expression, tumor mutational burden, and DNA mismatch repair deficiency as biomarkers for anti-PD-1 therapy (Teng et al., 2018; Darvin et al., 2018; ElOsta \& Jafri, 2019). In particular, PDL1 expression—recommended by the National Comprehensive Cancer Network guidelines - is widely recognized as the most powerful predictive marker for immunotherapy in lung adenocarcinoma. Moreover, patients with PDL1positive lung adenocarcinoma are more likely to benefit from immunotherapy than those with PDL1-negative lung adenocarcinoma (Gridelli et al., 2018; Dafni et al., 2019), suggesting that PDL1 expression plays a major role in the pathogenesis of lung adenocarcinoma. However, the mechanism underlying this clinical problem remains unclear. Understanding the regulatory role of PDL1 expression in lung adenocarcinoma is crucial for precision immunotherapy. Currently, PDL1 expression is primarily determined using immunohistochemistry (IHC) assays; nevertheless, IHC assays yield inconsistent results due to variable cutoffs and different antibodies with differing affinities (Hunter, Socinski, \& Villaruz, 2018). Hence, such assays cannot comprehensively elucidate the molecular mechanism and enriched pathways underlying the efficacy of immunotherapy. To solve this problem, novel strategies are imperative to explore the intrinsic mechanism of PDL1 expression in the biology of lung adenocarcinoma. Because of advances in next-generation sequencing technology, high-throughput sequencing results on cancers have been increasingly released on the Gene Expression Omnibus (GEO), a public database; this can thus enable understanding the molecular mechanism of PDL1 
101

102

103

104

105

106

107

expression and pathogenesis of lung adenocarcinoma. Using next-generation sequencing technology, previous studies have successfully identified key biomarkers in lung adenocarcinoma, which has proved to be essential in understanding the molecular mechanism of tumors (Tang et al., 2018; Li et al., 2019). Because PDL1 expression might affect certain key pathways in immunotherapy for lung adenocarcinoma, exploring the differences between patients with PDL1-positive and those with PDL1-negative lung adenocarcinoma would provide insights into the regulatory mechanisms of immunotherapy. Hence, it is reasonable to explore the differences in gene expression profiles and enriched pathways between the two groups of patients. Using the GEO database, we studied a gene expression dataset (GEO accession number: GSE99995) and comparatively analyzed sequencing data of gene expression between patients with PDL1-positive and PDL1-negative lung adenocarcinoma using the Agilent oligonucleotide microarray system. The aim of the study was to explore the differences in gene expression profiles and enriched pathways between the two groups of patients and identify the potential biomarkers predicting patient prognosis in order to elucidate the regulatory mechanism of PDL1 in immunotherapy, which could be crucial in guiding precision immunotherapy and improve prognosis in lung adenocarcinoma.

\section{MATERIAL AND METHODS}

\section{Gene expression profile data}

The GEO database is a public functional genomics data repository

(https://www.ncbi.nlm.nih.gov/geo/). We obtained gene expression profile data from the public 
121 GEO database based on the keywords "PDL1 expression," "lung adenocarcinoma," and "homo

122 sapiens." GSE99995 was retrieved. To prevent interferon expression levels having effects on

123 PDL1 gene expression profiles, only patients with lung adenocarcinoma with low interferon

124 expression levels were included. Patients with and without PDL1 expression were divided into

125 PDL1-positive (3 people, average age: $52.33 \pm 4.04$ years, tumor stage IIIA) and PDL1-negative

126 (3 people, average age: $51.33 \pm 3.06$ years, tumor stage IIIA) groups, respectively.

\section{DEGs analysis and mapping}

128 A series of matrix files and platforms were downloaded and processed using R. Screening of differentially expressed genes (DEGs) between the two groups as well as heat mapping and volcano mapping were performed using Limma. A log FoldChange of $>2$ and an adjusted $\mathrm{P}$ value of $<0.05$ indicated the presence of DEGs.

Gene Ontology and Kyoto Encyclopedia of Genes and Genomes pathway enrichment analysis

We used Gene Ontology (GO) and Kyoto Encyclopedia of Genes and Genomes (KEGG) pathway enrichment analyses to understand gene functional annotation and functional enrichment, respectively. We used the Database for Annotation, Visualization and Integrated

Discovery (DAVID) to perform GO and KEGG annotation of DEGs. DAVID is an online implications. Fisher's exact test was employed for analyses of pathways, diseases, and functions. 
140 A P value of $<0.05$ is recommended because it denotes the significance of GO terms and KEGG

141 pathway enrichment in genes. The top 10 GO terms and KEGG pathway enrichment results were

142 mapped using Hmisc and ggplot2 in R.

\section{Gene set enrichment analysis pathway enrichment analysis and validation}

144 Gene Set Enrichment Analysis (GSEA) is a computational method-based on the analysis of all

145 genes - that determines whether an a priori defined set of genes shows statistically significant

146 differences between two biological states. In the KEGG pathway analysis, the threshold value

147 was set according to the expression level, which could only be analyzed in genes with

148 significantly different expression levels. To avoid limitations in our results, GSEA was used

149 again to consider the effect of all DEGs, not limited to those with significantly different

150 expression levels. Therefore, GSEA was used to comprehensively analyze the differences in

151 gene pathway enrichment results between the two groups.

152 Protein-protein interaction network and analysis of hub genes

153 STRING is an online database designed to evaluate and predict protein-protein interactions

154 (PPIs) (https://string-db.org/cgi/input.pl). First, STRING was used in this study to analyze the

155 PPI network of different genes in the two patient groups. Isolated nodes were removed, and the

156 results of the interaction network were downloaded and then imported into Cytoscape (version

157 3.7.2) for subsequent analysis. Second, the PPI network was constructed and visualized using

158 Cytoscape (version 3.7.2) and cytoHubba. The MNC algorithm was employed to screen and 
159

160

161

162

163

164

165

166

167

168

169

170

171

172

173

174

175

identify the hub genes that might be key candidate genes with crucial regulatory functions.

\section{Clinical characteristics and survival analysis related to hub genes}

Gene Expression Profiling Interactive Analysis (GEPIA) is an online cancer data mining site that is based on the TCGA and GTEx databases and uses a standard processing pipeline (http://gepia.cancer-pku.cn/detail.php). In this study, the online survival analysis tool of GEPIA was used to verify our previous results obtained using the TCGA and GTEx databases. Furthermore, differences in the expression of hub genes in lung adenocarcinoma and adjacent normal tissues were analyzed. Moreover, the relationships among differences in expression of hub genes, pathological staging, and prognosis in lung adenocarcinoma were analyzed. Furthermore, $\mathrm{P}$ values of $<0.05$ were considered statistically significant.

\section{Mutation characteristics and pathway analyses of hub genes}

We investigated the mutation characteristics and possible functional mechanisms of the differential expression of hub genes in lung adenocarcinoma. Therefore, data sets containing gene expression profiles of patients with lung adenocarcinoma were included in the TCGA database (e.g., Broad, Cell 2012; MSKCC, Science [2015]; TCGA, Firehose Legacy; TCGA, Nature [2014]; and TCGA, PanCancer Atlas). A combined study of five data sets including 1598 patients and 1600 samples were included in the analysis.

GSCALite is a web-based analysis platform that analyzes an entire set of genes in cancers (http://bioinfo.life.hust.edu.cn/web/GSCALite/), including alterations in DNA or RNA of cancer- 
178

179

180

181

182

183

184

related genes, the activity of 10 cancer-related pathways, and miRNA regulatory network for genes. To understand the cancer-related pathways of hub genes, we distinguished hub gene expression between pathway activation and inhibition groups according to pathway scores in GSCALite.

\section{RESULTS}

\section{Identification and mapping of DEGs}

Our results revealed that the PDL1-positive and PDL1-negative groups comprised 34,729 genes. According to criteria of a $\log$ FoldChange of $>2$, and an adjusted $\mathrm{P}$ value of $<0.05$, a total of 869 DEGs were identified, with 387 and 482 DEGs in the PDL1-positive and PDL1-negative groups, respectively. The corresponding heat map and volcano map are shown in Figure 1.

\section{GO Terms and KEGG pathway enrichment analysis of DEGs}

The analysis of DEGs by using GO Terms was divided into three parts, namely biological processes, cellular components, and molecular functions. The GO analysis of the PDL1-positive group mainly revealed the enrichment of pathways involved in cell adhesion (BP, GO:0007155) plasma membrane (CC, GO:0005886), and cadmium ion binding (MF, GO:0046870). The GO analysis of the PDL1-negative group mainly revealed the enrichment of pathways involved in cell division (BP, GO:0051301), nucleoplasm (CC, GO:0005654), and DNA binding (MF,GO:0003677). The detailed results are shown in Table 1. The KEGG pathway enrichment results in the PDL1-positive group revealed that the enriched pathways were mainly those 
197 198 199

involved in cell adhesion molecules, PPAR signaling, and ECM-receptor interaction. However, the KEGG pathway enrichment results of the PDL1-negative group revealed that the enriched pathways were mainly those involved in DNA replication, cell cycle, and mismatch repair (Table 2). The detailed results are shown in Table 2. The top 10 GO terms and KEGG enriched pathways were mapped using the Hmisc and ggplot2 packages. The results are shown in Figure 2.

\section{GSEA enrichment analysis and validation}

The results were validated through GSEA analysis after all the DEGs were considered. The GSEA results also provided the KEGG results. However, a new pathway enrichment was observed in the PDL1-positive group, namely the T-cell receptor pathway, which was not observed in KEGG results of DEGs (Fig. 3).

\section{Construction of PPI network and analysis of hub genes}

The STRING database consists of known and predicted PPIs. The interactions include direct (physical) and indirect (functional) associations. To explore the expression relationships between the DEGs, a PPI network was constructed in our study. Our results showed that the PPI network consisted of 570 nodes and 2937 edges (Fig. 4A). The average node degree was 10.3 and the average local clustering coefficient was 0.404 (PPI enrichment $\mathrm{p}$ value: $<1.0 \mathrm{e}-16$ ). In addition, the MNC algorithm was used to screen and identify hub genes that might be key genes with crucial regulatory functions. The top 10 hub genes, BUB1B, CDC45, BUB1, TTK, BRCA1, TOP2A, NDC80, RFC4, MCM2, and DTL, were identified in our study (Fig. 4B). The results 

were ranked using the MNC method, as shown in Table 3.

\section{Analysis of the mechanism of hub genes in the TCGA database}

218 To prevent the limitations caused by using a single GEO database, our results were verified using

219 the TCGA and GTEx databases. First, comparative analysis of hub gene expression in lung 220 adenocarcinoma and adjacent normal tissue was performed in our study, and our results showed

221 that the expression levels of BUB1B, CDC45, BUB1, TTK, TOP2A, NDC80, MCM2, and DTL

222 were significantly higher in lung adenocarcinoma tissues than in adjacent normal lung tissues (P

$223<0.01)$. However, although the expression levels of BRCA1 and RFC4 were higher in lung

224 adenocarcinoma tissues than in adjacent normal tissues, the differences were not significant (Fig. 225 5).

226 In addition, the relationship between hub genes and pathological staging were analyzed. The

227 results showed that $\mathrm{BUB} 1 \mathrm{~B}(\mathrm{~F}$ value $=5.22, \mathrm{p}=0.00148), \mathrm{CDC} 45(\mathrm{~F}$ value $=2.86, \mathrm{P}=0.0364)$,

228 BUB1 $(F$ value $=5.22, \mathrm{P}=0.00149), \mathrm{TTK}(\mathrm{F}$ value $=5.06, \mathrm{P}=0.00185), \mathrm{BRCA} 1(\mathrm{~F}$ value $=4.9$,

$229 \mathrm{P}=0.0023)$, TOP2A $(\mathrm{F}$ value $=2.88, \mathrm{P}=0.0354), \mathrm{NDC} 80(\mathrm{~F}$ value $=3.58, \mathrm{P}=0.014), \mathrm{MCM} 2(\mathrm{~F}$

230 value $=2.78, \mathrm{P}=0.0407)$, and DTL $(\mathrm{F}$ value $=5.97, \mathrm{P}=0.000535)$ were positively correlated

231 with pathological staging, and the differences were statistically significant. However, regarding

232 the correlation between RFC4 and pathological staging, although RFC4 was positively correlated

233 with pathological staging, the expression of RFC4 did not differ significantly between the two

234 groups $(\mathrm{F}$ value $=1.82, \mathrm{P}=0.142 ;$ Fig. 6). 
235 Finally, the relationship between the hub genes and prognosis was analyzed. The results showed

236 that patients with lung adenocarcinoma with low expression levels of BUB1B $(\log \operatorname{rank} \mathrm{P}=$

$2373.8 \mathrm{e}-05, \mathrm{P}(\mathrm{HR})=5.1 \mathrm{e}-05), \mathrm{CDC} 45(\log \operatorname{rank} \mathrm{P}=0.0032, \mathrm{p}(\mathrm{HR})=0.0035), \mathrm{BUB} 1(\log \operatorname{rank} \mathrm{P}$

$238=0.0024, \mathrm{p}(\mathrm{HR})=0.0026), \mathrm{TTK}[\log \operatorname{rank} \mathrm{P}=0.00029, \mathrm{P}(\mathrm{HR})=0.00034], \mathrm{BRCA} 1[\log \operatorname{rank}$

$239 \mathrm{P}=0.0035, \mathrm{P}(\mathrm{HR})=0.0038]$, TOP $2 \mathrm{~A}[\log \operatorname{rank} \mathrm{P}=0.011, \mathrm{P}(\mathrm{HR})=0.012], \mathrm{NDC} 80[\log \operatorname{rank} \mathrm{P}$

$240=0.0027, \mathrm{P}(\mathrm{HR})=0.003], \mathrm{MCM} 2[\log \operatorname{rank} \mathrm{P}=0.02, \mathrm{P}(\mathrm{HR})=0.021]$, and $\mathrm{DTL}[\log \operatorname{rank} \mathrm{P}=$

$2410.0016, \mathrm{P}(\mathrm{HR})=0.0018]$ had significantly higher overall survival than those with high gene

242 expression levels $(\mathrm{P}<0.05)$. However, no significant correlation was observed between RFC4

243 gene expression level and overall survival (log rank $\mathrm{P}=0.059$; Fig. 7).

\section{Analysis of the mechanism of hub genes in the TCGA database}

245 Furthermore, analysis of the lung adenocarcinoma was performed using the database TCGA. The

246 results showed that the hub genes had different mutation frequencies, ranging from $1 \%$ to $5 \%$.

247 For example, BUB1B (4\%), CDC45 (1.9\%), and DTL (5\%) (Fig. 8A). Moreover, the potential

248 hub genes exhibited different mutation forms in lung adenocarcinoma, which may facilitate

249 further exploration of the function of these hub genes in lung adenocarcinoma. For example,

250 BUB1B mainly exhibited deep deletion, whereas BRCA1 mainly exhibited amplification (Fig.

251 8B). However, these different mutant forms of hub genes were involved in different signaling

252 pathways influencing the development of lung adenocarcinoma; for example, $B R C A 1$ was

253 mainly involved in the cell cycle and DNA damage response pathways (Fig. 9A, B).

\section{DISCUSSION}


255 Lung adenocarcinoma is a highly heterogeneous type of cancer, and its individualized treatment

256 has attracted considerable attention. An increasing number of studies have recently indicated that

257 immunotherapy, especially anti-PD-1 therapy, is a promising strategy for treating lung

258 adenocarcinoma. Some anti-PD-1 antibodies have been successfully commercialized and

259 approved as first- and second-line immunotherapy options for advanced NSCLC in the past few

260 years. Nevertheless, the overall response rate for such immunotherapy is only approximately

$26120 \%$ (Greillier, Tomasini, \& Barlesi, 2018), and the efficacy of such immunotherapy is affected

262 by PDL1 expression. In particular, PDL1 expression has been proposed as a predictive

263 biomarker, suggesting that it plays a major role in immune regulation in lung adenocarcinoma.

264 Therefore, understanding the effects of PDL1 expression on the biological behavior and the

efficacy of immunotherapy in lung adenocarcinoma is crucial. However, the use of IHC alone to

assess PDL1 expression cannot provide a complete explanation for the molecular mechanism and

enriched pathways underlying the efficacy of immunotherapy. With the development of next-

generation sequencing technology and bioinformatics, a novel strategic solution to this problem

may be identified (Morganti et al., 2019). Furthermore, previous studies have used bioinformatic

analysis to explore the core genes of lung adenocarcinoma and their malignant transformation

mechanisms (Yuan et al., 2017; Yeh et al., 2019). However, no research has been conducted on

PDL1-positive and PDL1-negative patients. Understanding DEGs and differences in the

biological processes and enrichment pathways between PDL1-positive and PDL1-negative

patients is crucial. Hence, by using gene bioinformatics analysis, we explored the differences in 
276 identified the potential key biomarkers that could be used for predicting disease prognosis in

277 patients. The elucidation of the regulatory mechanism of PDL1 is crucial to improve precision

278 immunotherapy for lung adenocarcinoma.

279 Our study demonstrated that even in patients with lung adenocarcinoma, differences in gene

280 expression were observed between those in the PDL1-positive and PDL1-negative group, thus

281 suggesting that these two types of patients should be treated differently.

282 The important findings in our study in the GO Terms and KEGG pathway analysis were the

283 differences between the PDL1-positive and PDL1-negative groups of patients with lung

284 adenocarcinoma. Our results reveal that the PDL1-positive group mainly demonstrated

285 enrichment of adhesion-related biological processes and the PPAR signaling pathway. Adhesion-

286 related biological processes have been previously reported to be associated with invasion and

287 metastasis in patients with lung adenocarcinoma (Stevens et al., 2017; Alonso-Nocelo et al.,

288 2018), resulting in relatively high levels of organ and tissue metastases and poor prognosis. Real-

289 world studies have revealed similar results (Wang et al., 2015; Okita et al., 2017). Previous

290 studies have reported that PPAR, an anti-inflammatory molecule with a potent, could promote

291 tumor proliferation, angiogenesis, inflammation, and metastasis in lung adenocarcinoma (Reka et

292 al., 2011; Ammu et al., 2019), suggesting that it is a vital and potential therapeutic target for lung

293 adenocarcinoma. Moreover, Lv and Wang (2015) discovered that the PPAR signaling pathway

294 played a major role in malignant transformation of cells among nonsmoking patients with lung

295 adenocarcinoma. These results are consistent with our findings. Although several studies have 
296

297

298

299

300

301

302

303

304

305

306

307

308

309

310

311

312

313

314

315

suggested the relationship between PPAR and lung adenocarcinoma, the role of PPAR in

immunotherapy for lung adenocarcinoma has yet to be reported. Moreover, recent studies have

demonstrated that PPAR could enhance indoleamine 2,3-dioxgenase-1 (IDO) activity and

promote the generation of regulatory T cells in melanoma; hence, the use of PPAR inhibitors

could enhance cancer immunotherapy (Poupot et al., 2014). On the basis of our results along

with the results of these studies, we hypothesized that the PPAR pathway might play a crucial

role in immunotherapy for lung adenocarcinoma. A comprehensive analysis of our results

indicated that although PDL1 expression was associated with the immune response pathway, it

might have potential to contribute to invasion and metastasis in patients with PDL1-positive lung

adenocarcinoma.

Conversely, the results of the PDL1-negative group mainly revealed enrichment of division-

related biological processes and repair systems pathways, which indicated that these pathways

might be able to repair defects in apoptosis or recombination to reduce the production of

neoantigens. Some clinical studies have shown that PDL1-negative patients tend to have lower

tumor mutation burdens or fewer neoantigens than PDL1-positive patients (Xia et al., 2017;Chan

et al., 2019), which might be related to its involvement in various repair systems. Further

research must be conducted to confirm our conclusions.

In addition, the GSEA results were similar to those for the KEGG pathway analysis. More

importantly, a notable and novel finding was reported in the PDL1-positive group, namely that

related to the T-cell receptor pathway, which has been proved to be crucial for achieving a 
316 favorable immunotherapeutic response in NSCLC (Van De \& Borst, 2015). This finding

317 suggests that PDL1 might be involved in the regulation of the T-cell pathway; therefore,

318 understanding the effects of PDL1 expression on tumor microenvironmental immune cells and

319 mediation of T-cell pathways is necessary.

320 In our study, 10 hub genes screened in the GEO database were verified and further analyzed

321 using the TCGA and GTEx databases. Our study further analyzed the prognostic value and

322 possible mechanism of hub genes in lung adenocarcinoma considering their importance.

323 Several hub genes, such as BUB1B, CDC45, BUB1, TTK, TOP2A, MCM2, NDC80, and DTL,

324 have been reported to be associated with poor prognosis in patients with lung adenocarcinoma

325 (Hayama et al., 2006; He et al., 2019; Liu et al., 2017; Perez-Peña et al., 2017; Song et al., 2018;

326 Sun et al., 2020). Our findings corroborate these results. Another major finding of our study is

327 that BRCA1 is a predictor in patients with lung adenocarcinoma.

328 BRCA1 encodes a nuclear phosphoprotein that helps maintain genomic stability. It is involved in

329 biological processes such as cell cycle regulation, replication, and mitotic spindle assembly.

330 Previous studies have suggested that BRCA1 overexpression regulates drug response in

331 chemotherapy, is related to the efficacy of EGFR-TKIs, and is prevalent in patients with NSCLC

332 with early disease onset (Reguart et al., 2008; Sun et al., 2018). Recent studies have analyzed the

333 potential prognostic role of BRCA1 in early-stage NSCLC, suggesting that BRCA1 is a predictor

334 of survival in only stage III NSCLC (Hu et al., 2019). Although several studies have

335 demonstrated the role of BRCA1 in NSCLC, the prognostic value and possible mechanism of 
336

337

338

339

340

341

342

343

344

345

346

347

348

349

350

351

352

353

354

355

BRCA1 in lung adenocarcinoma remain unclear. Our study results suggest that BRCA1

overexpression was associated with poor prognosis in lung adenocarcinoma. In particular, our results indicate that BRCA1 was mainly involved in cell cycle and DNA damage responses in lung adenocarcinoma, which might produce new antigens and enhance immune responses.

BRCA1 mutation was also reported to be associated with tumor neoantigen production, immune cell invasion, and PDL1 expression in ovarian cancer (Strickland et al., 2016). Overall, these findings suggest that BRCA1 plays a key role in the immunomodulatory pathway of lung adenocarcinoma.

RFC4, also named activator 1, is a protein complex consisting of five subunits measuring 140, 40, 38, 37, and 36 kD. Telomere C-strand (Lagging Strand) Synthesis and E2F-mediated regulation of DNA replication are some of its related pathways. A previously conduced weighted gene coexpression network analysis revealed that it was a prognosis-related biomarker in lung adenocarcinoma (Yi et al., 2020). However, our GEPIA survival analysis showed that RFC4 overexpression was positively correlated with pathological staging but was not associated with poor prognosis in patients with lung adenocarcinoma. Possible reasons for these inconsistent findings are differences in sample selection strategies and algorithms. Accordingly, the prognostic value of RFC4 for lung adenocarcinoma warrants further study.

The present study has several limitations. First, only a single GSE was searched and analyzed in 54 the GEO database. To prevent bias resulting from the analysis of a single data set, we adopted an integrated bioinformatic analysis approach. Second, the main objective of this study was to 
356

357

358

359

360

361

362

363

364

explore the influence of PDL1 expression on related gene expression profiles in patients with lung adenocarcinoma. Hence, this study was divided into expression in PDL1-positive and PDL1-negative groups; however, no further distinction was made in the PDL1-positive group between patients with high (50\%) and low $(<1 \%)$ levels of PDL1 expression. Different PDL1 expression levels might have caused differences in the expression profiles of genes, which warrants further confirmation. Third, some key genes have been studied in various tumors, including non-small-cell lung cancer. However, this study identified core genes that might be related to or regulated by the expression of PDL1 in lung adenocarcinoma, which will assist in the further discovery of mechanisms for designing novel immunotherapeutic options.

This study revealed the DEGs and different biological pathways between PDL1-positive and PDL1-negative patients with lung adenocarcinoma, speculating that these two types of patients might have different subtypes of lung adenocarcinoma. The identification and verification of hub genes through integrated bioinformatic analysis revealed that they related to immune response pathways and prognosis in patients with lung adenocarcinoma. In particular, they had different mutations and were involved in different pathways in lung adenocarcinoma.

\section{CONCLUSIONS}

PDL1-positive lung adenocarcinomaand PDL1-negative lung adenocarcinoma might be different subtypes of lung adenocarcinoma. Potential hub genes might be involved in PDL1 regulatory pathways, and further research is warranted to reveal new mechanisms underlying the regulation of PDL1 expression. This could be of great significance for precision immunotherapy for lung adenocarcinoma. 
376

377 378

379 380

381

382

383

384

385

386

387

388

389

390

391

392

393

394

395

396

397

398

399

400

401

402

403

\section{REFERENCES}

1.Herbst R S , Morgensztern D, Boshoff C. 2018. The biology and management of non-small cell lung cancer. Nature 553: 446-454 DOI: 10.1038/nature25183.

2.Zhang L, Peng R, Sun Y, Wang J, Chong X, Zhang Z. 2019. Identification of key genes in non-small cell lung cancer by bioinformatics analysis. Peer J 7:e8215 DOI: 10.7717/peerj.8215.

3.Singhi E K, Horn L, Sequist L V, Heymach J, Langer C J. 2019. Advanced Non-Small Cell Lung Cancer: Sequencing Agents in the EGFR-Mutated/ALK-Rearranged Populations. American Society of Clinical Oncology Educational Book 39: e187-e197 DOI: 10.1200/EDBK_237821.

4.Sgambato A, Casaluce F, Maione P, Gridelli C. 2018. Targeted therapies in non-small cell lung cancer: a focus on ALK/ROS1 tyrosine kinase inhibitors. Expert review of anticancer therapy 18: 71-80 DOI: $10.1080 / 14737140.2018 .1412260$.

5.Blandin Knight S, Crosbie PA, Balata H, Chudziak J, Hussell T, Dive C. 2017. Progress and prospects of early detection in lung cancer. Open biology 7: 170070 DOI: 10.1098/rsob.170070.

6.Yu X, Wang X. 2018. Tumor immunity landscape in non-small cell lung cancer. Peer J 6:e4546 DOI: $10.7717 /$ peerj.4546.

7.Lorigan P, Eggermont AMM. 2019. Anti-PD1 treatment of advanced melanoma: development of criteria for a safe stop. annals of oncology 30:1038-1040 DOI: 10.1093/annonc/mdz182.

8.Morse MA, Hochster H, Benson A. 2020. Perspectives on Treatment of Metastatic Colorectal Cancer with Immune Checkpoint Inhibitor Therapy. Oncologist 25:33-45 DOI: 10.1634/theoncologist.2019-0176.

9. El-Osta H, Jafri S. 2019. Predictors for clinical benefit of immune checkpoint inhibitors in advanced non-smallcell lung cancer: a meta-analysis. Immunotherapy 11:189-199 DOI: 10.2217/imt-2018-0086.

10.Dafni U, Tsourti Z, Vervita K, Peters S. 2019. Immune checkpoint inhibitors, alone or in combination with chemotherapy, as first-line treatment for advanced non-small cell lung cancer. A systematic review and network meta-analysis. Lung Cancer 134:127-140 DOI: 10.1016/j.lungcan.2019.05.029.

11.Gridelli C, Ascierto PA., Grossi F, Baldini E, Favaretto A, Garassino MC, Morabito A,Migliorino M R, Rossi A, de Marinis F. 2018. Second-line treatment of advanced non-small cell lung cancer non-oncogene addicted: New treatment algorithm in the era of novel immunotherapy. Current clinical pharmacology 13: 76-84 DOI: $10.2174 / 1574884713666180711160008$.

Peer) reviewing PDF | (2020:02:45804:2:0:NEW 4 May 2020) 
404 12.Suresh K, Naidoo J, Lin CT, Danoff S. 2018. Immune checkpoint immunotherapy for non-small cell lung cancer:

405 benefits and pulmonary toxicities. Chest 154: 1416-1423 DOI: 10.1016/j.chest.2018.08.1048.

406 13.Teng, F, Meng, X., Kong, L, Yu J, 2018. Progress and challenges of predictive biomarkers of anti PD-1/PD-L1

407 immunotherapy: a systematic review. Cancer letters 414:166-173 DOI: 10.1016/j.canlet.2017.11.014.

408 14.Darvin, P, Toor, SM, Nair, VS, Elkord E. 2018. Immune checkpoint inhibitors: recent progress and potential

409 biomarkers. Experimental \& molecular medicine 50:1-11 DOI: 10.1038/s12276-018-0191-1.

410 15.Hunter KA, Socinski MA, Villaruz LC. 2018. PD-L1 testing in guiding patient selection for PD-1/PD-L1

411 inhibitor therapy in lung cancer. Molecular diagnosis \& therapy 22:1-10 DOI: 10.1007/s40291-017-0308-6.

412 16.Li Z, Sang M, Tian Z, Liu Z, Lv J, Zhang F, Shan B. 2019. Identification of key biomarkers and potential

413 molecular mechanisms in lung cancer by bioinformatics analysis. Oncology letters 18: 4429-4440

414 DOI: 10.3892/ol.2019.10796.

415 17.Tang Q, Zhang H, Kong M, Mao X, Cao X. 2018. Hub genes and key pathways of non-small lung cancer 416 identified using bioinformatics. Oncology letters 16: 2344-2354 DOI: 10.3892/ol.2018.8882.

417 18.Greillier L, Tomasini P, Barlesi F. 2018. The clinical utility of tumor mutational burden in non-small cell lung 418 cancer. Translational lung cancer research. 7:639-646 DOI: 10.21037/tlcr.2018.10.08.

419 19.Morganti S, Tarantino P, Ferraro E, D’Amico P, Duso BA, Curigliano G. 2019. Next Generation Sequencing 420 (NGS): A Revolutionary Technology in Pharmacogenomics and Personalized Medicine in Cancer. Advances in 421 Experimental Medicine and Biology 1168: 9-30 DOI: 10.1007/978-3-030-24100-1_2.

422 20. Yuan J, Zhang N, Yin L, Zhu H, Zhang L, Zhou L, Yang M. 2017. Clinical implications of the autophagy core 423 gene variations in advanced lung adenocarcinoma treated with gefitinib. Scientific reports 7: 1-10

424 DOI: 10.1038/s41598-017-18165-5.

425 21.Yeh SJ, Chang CA, Li CW, Wang LH, Chen BS. 2019. Comparing progression molecular mechanisms between 426 lung adenocarcinoma and lung squamous cell carcinoma based on genetic and epigenetic networks: big data

427 mining and genome-wide systems identification. Oncotarget 10:3760-3806.DOI: 10.18632/oncotarget.26940.

428 22.Stevens LE, Cheung WK, Adua SJ, Arnal-Estapé A, Zhao M, Liu Z, Brewer K, Herbst RS, Nguyen DX. 2017.

429 Extracellular matrix receptor expression in subtypes of lung adenocarcinoma potentiates outgrowth of

430 micrometastases. Cancer research 77: 1905-1917 DOI: 10.1158/0008-5472.CAN-16-1978.

431 23.Alonso-Nocelo M, Raimondo TM, Vining KH, López-López R, de la Fuente M, Mooney DJ. 2018. Matrix

432 stiffness and tumor-associated macrophages modulate epithelial to mesenchymal transition of human

433 adenocarcinoma cells. Biofabrication 10: 035004 DOI: 10.1088/1758-5090/aaafbc.

Peer) reviewing PDF | (2020:02:45804:2:0:NEW 4 May 2020) 
434 24.Okita R, Maeda A, Shimizu K, Nojima Y, Saisho S, Nakata M. 2017. PD-L1 overexpression is partially regulated 435 by EGFR/HER2 signaling and associated with poor prognosis in patients with non-small-cell lung cancer. Cancer 436 Immunology, Immunotherapy 66: 865-876 DOI: 10.1007/s00262-017-1986-y.

437 25.Wang A, Wang HY, Liu Y, Zhao MC, Zhang HJ, Lu ZY, Fang YC, Chen XF,Liu GT. 2015. The prognostic 438 value of PD-L1 expression for non-small cell lung cancer patients: a meta-analysis. European Journal of Surgical 439 Oncology 41: 450-456 DOI: 10.1016/j.ejso.2015.01.020.

440 26.Ammu VRK, Garikapati KK, Krishnamurthy PT, Chintamaneni PK, Pindiprolu SKS. 2019. Possible role of 441 PPAR- $\gamma$ and COX-2 receptor modulators in the treatment of Non-Small Cell lung carcinoma. Medical hypotheses 442 124: 98-100 DOI: 10.1016/j.mehy.2019.02.024.

27.Reka AK, Goswami MT, Krishnapuram R, Standiford TJ, Keshamouni VG. 2011. Molecular cross-regulation between PPAR- $\gamma$ and other signaling pathways: implications for lung cancer therapy. Lung cancer 72:154-159 DOI: 10.1016/j.lungcan.2011.01.019.

446 28.Lv, M., \& Wang L. 2015. Comprehensive analysis of genes, pathways, and TFs in nonsmoking Taiwan females 447 with lung cancer. Experimental lung research 41:74-83 DOI: 10.3109/01902148.2014.971472.

448 29.Poupot M, Boissard F, Betous D, Bardouillet L, Fruchon S, L'Faqihi-Olive F, Mekaouche M, Ingoure S, Sicard 449 H, Dubreuilh G, Fournié JJ. 2014. The PPAR $\alpha$ pathway in V $\gamma 9$ V82 T cell anergy. Cellular and Molecular Biology 450 Letters 19: 649-658 DOI: 10.2478/s11658-014-0218-0.

451 30.Xia H, Shen J, Hu F, Chen S, Huang H, Xu Y, Ma H. 2017. PD-L1 over-expression is associated with a poor 452 prognosis in Asian non-small cell lung cancer patients. Clinica Chimica Acta 469: 191-194

453 DOI: 10.1016/j.cca.2017.02.005.

454 31.Chan TA, Yarchoan M, Jaffee E, Swanton C, Quezada SA, Stenzinger A, Peters S. 2019.Development 455 of tumor mutation burden as an immunotherapy biomarker: utility for the oncology clinic. Annals of Oncology 30 : 456 44-56 DOI: 10.1093/annonc/mdy495.

457 32.Van De Ven K, Borst J. 2015. Targeting the T-cell co-stimulatory CD27/CD70 pathway in cancer 458 immunotherapy: rationale and potential. Immunotherapy 7: 655-667 DOI: 10.2217/imt.15.32.

459 33.Song YJ, Tan J, Gao XH, Wang LX. 2018. Integrated analysis reveals key genes with prognostic value in lung 460 adenocarcinoma. Cancer management and research 10: 6097 DOI: 10.2147/CMAR.S168636.

461 34.He W, Fu L, Yan Q, Zhou Q, Yuan K, Chen L, Han Y. 2019. Gene set enrichment analysis and meta-analysis 462 identified 12 key genes regulating and controlling the prognosis of lung adenocarcinoma. Oncology letters 17: 56084635618 DOI: 10.3892/ol.2019.10236. 
464 35.Hayama S, Daigo Y, Kato T, Ishikawa N, Yamabuki T, Miyamoto M, Tsuchiya E, Kondo S, Nakamura Y. 2006.

465 Activation of CDCA1-KNTC2, members of centromere protein complex, involved in pulmonary carcinogenesis.

466 Cancer Research 66:10339-10348 DOI: 10.1158/0008-5472.CAN-06-2137.

467 36.Liu YZ, Wang BS, Jiang YY, Cao J, Hao JJ, Zhang Y,Xu X, Cai Y, Wang MR. 2017. MCMs expression in lung 468 cancer: implication of prognostic significance. Journal of Cancer 8: 3641 DOI: 10.7150/jca.20777.

469 37.Perez-Peña J, Corrales-Sánchez V, Amir E, Pandiella A, Ocana A. 2017. Ubiquitin-conjugating enzyme E2T

470 (UBE2T) and denticleless protein homolog (DTL) are linked to poor outcome in breast and lung cancers. Scientific 471 reports 7: 1-8 DOI: 10.1038/s41598-017-17836-7.

472 38.Sun ZY, Wang W, Gao H, Quan-Fang Chen QF.2020. Potential therapeutic targets of the nuclear division cycle 47380 (NDC80) complexes genes in lung adenocarcinoma J. Cancer 11: 2921-2934 doi:10.7150/jca.41834.

474 39.Reguart N, Cardona AF, Carrasco E, Gomez P, Taron M, Rosell R. 2008. BRCA1: A new genomic marker for 475 non-small-cell lung cancer. Clinical lung cancer 9: 331-339 DOI: 10.3816/CLC.2008.n.048.

40.Sun X, Cui F, Yin H, Wu D, Wang N, Yuan M, Fei Y, Wang Q. 2018. Association between EGFR mutation and 477 expression of BRCA1 and RAP80 in non-small cell lung cancer. Oncology letters 16:2201-2206

478 DOI: 10.3892/ol.2018.8938.

41.Hu X, Yang D, Li Y, Li L, Wang Y, Chen P, Xu S, Pu X, Zhu W, Deng P, Ye J, Zhang H, LIzaso A, Liu H, Mao X, Huang H, Chu Q, Hu C. 2019. Prevalence and clinical significance of pathogenic germline BRCA1/2 mutations in Chinese non-small cell lung cancer patients. Cancer Biology \& Medicine 16:556 DOI: 10.20892/j.issn.20953941.2018 .0506 .

42.Strickland KC, Howitt BE, Shukla SA, Rodig S, Ritterhouse LL, Liu JF, Matulonis UA, Konstantinopoulos PA. 2016. Association and prognostic significance of BRCA1/2-mutation status with neoantigen load, number of tumorinfiltrating lymphocytes and expression of PD-1/PD-L1 in high grade serous ovarian cancer. Oncotarget 7: 13587 DOI: $10.18632 /$ oncotarget. 7277.

43.Yi M, Li T, Qin S, Yu S, Chu Q, Li A, Wu K. 2020. Identifying Tumorigenesis and Prognosis-Related Genes of Lung Adenocarcinoma: Based on Weighted Gene Coexpression Network Analysis. BioMed Research International 4169691 DOI: 10.1155/2020/4169691. 


\section{Figure 1}

Heat map and volcanic plot of DEGs comparing patients with PDL1-positive and PDL1negative lung adenocarcinoma.

(A) Heat map of DEGs. (B) Volcanic plot of DEGs. Red represents high expression levels in the PDL1-positive group, whereas blue represents high expression levels in the PDL1-negative group. Gray represents no difference in expression levels between the two groups.

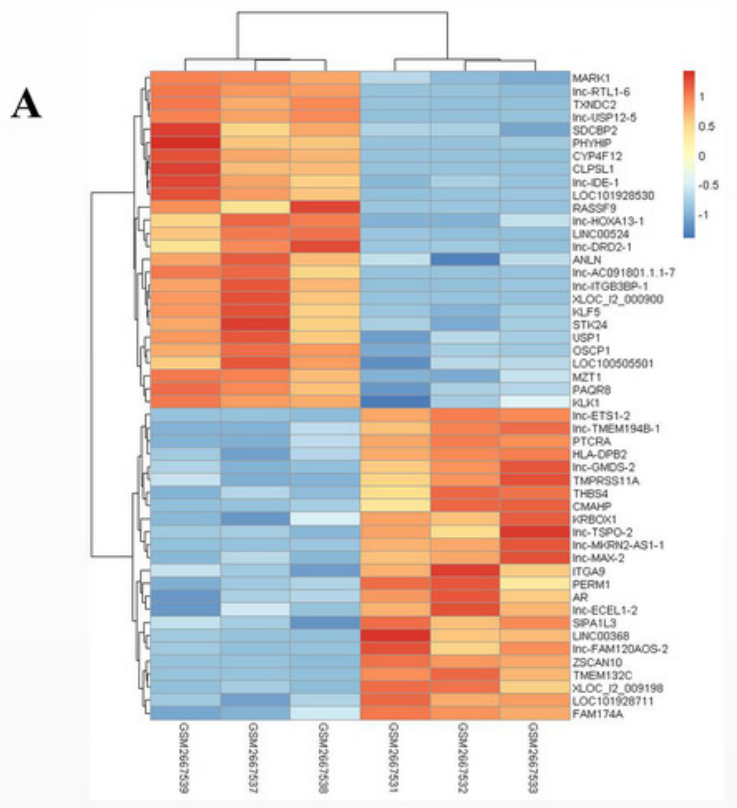

B

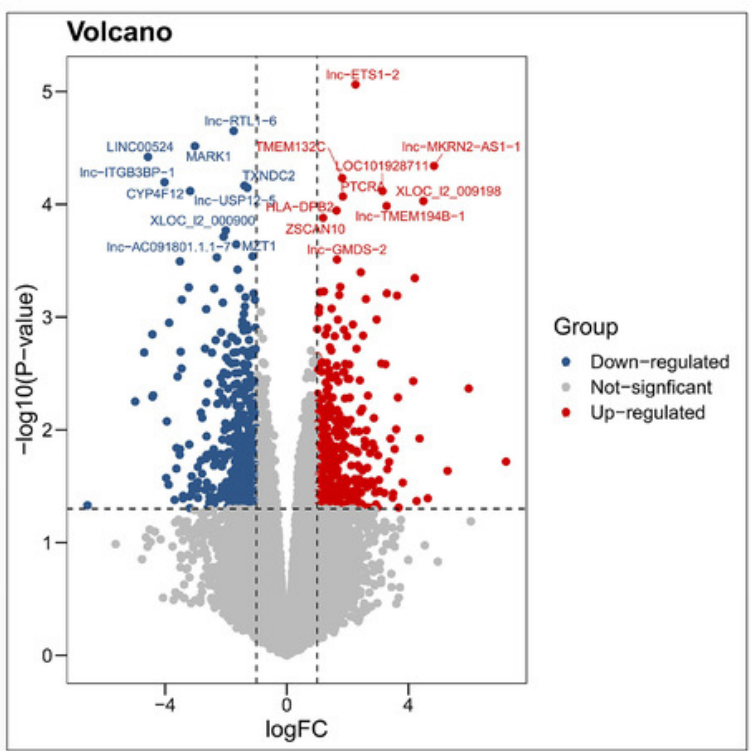




\section{Figure 2}

Bubble mapping of the top 10 GO Terms and KEGG pathway enrichment analysis data of DEGs

(A) GO analysis of DEGs in biological process. (B) GO analysis of DEGs in cellular components.

(C) GO analysis of DEGs in terms of molecular function. (D) KEGG enrichment analysis of DEGs. A high gene ratio represents a high level of enrichment. The size of the dot indicates the number of target genes in the pathway and the color of the dot reflects the $p$ value range. (E) GO Chord plot of DEGs. (F) KEGG Chord plot of DEGs. 
A

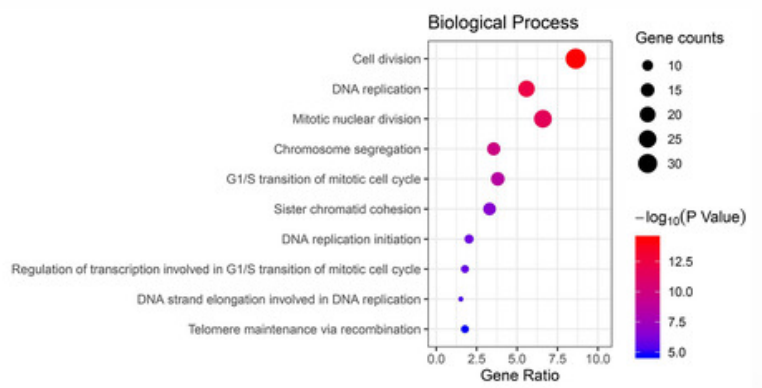

C

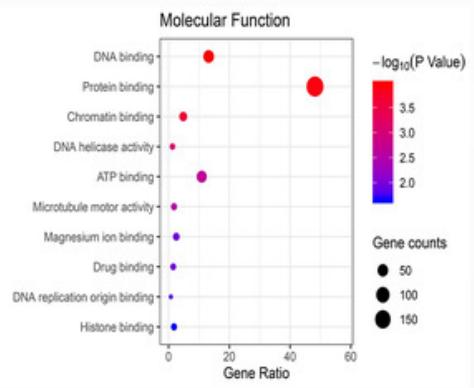

$\mathbf{E}$

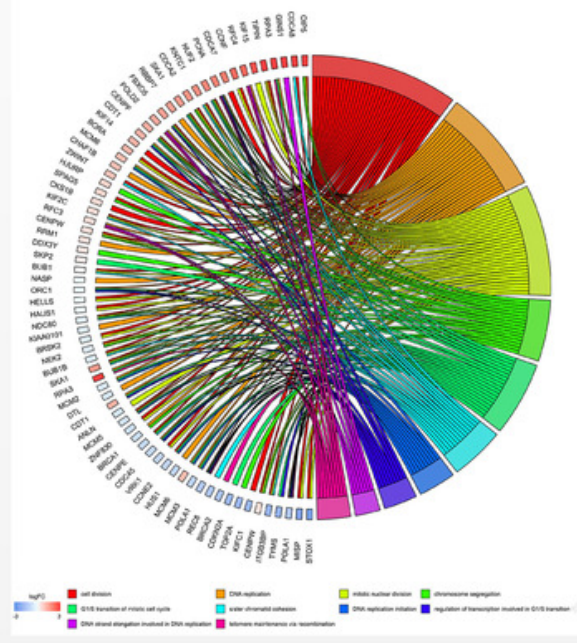

B

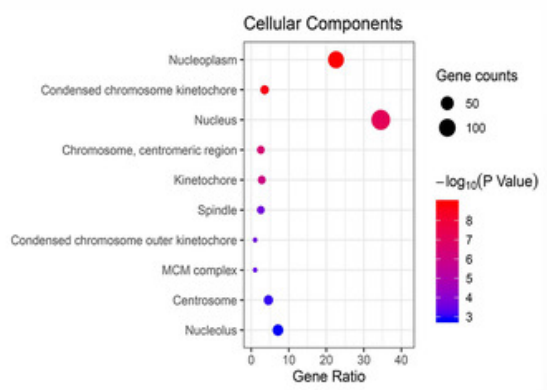

D

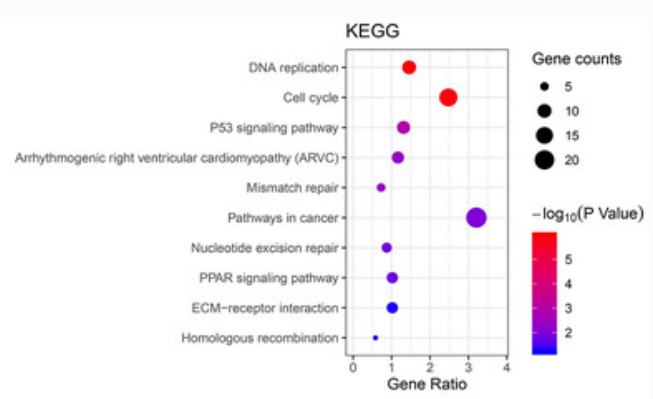

F

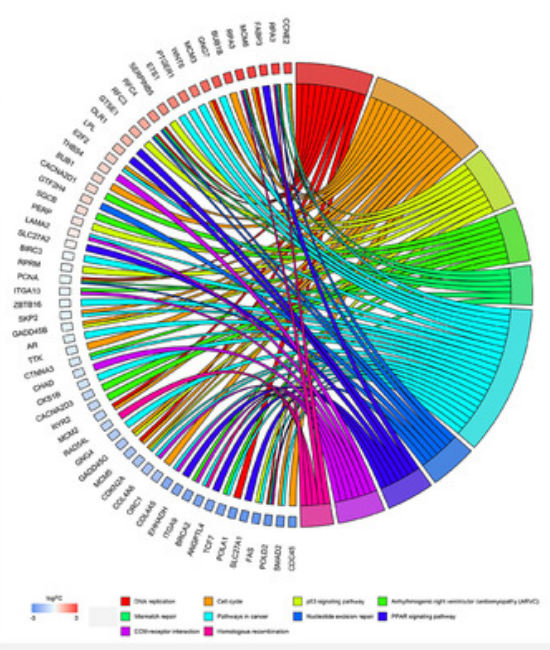


Figure 3

GSEA enrichment analysis and validation.

(A, B, C, D) GSEA enrichment analysis in the PDL1-positive group. (E, F, G, H) GSEA enrichment analysis in the PDL1-negative group .

A

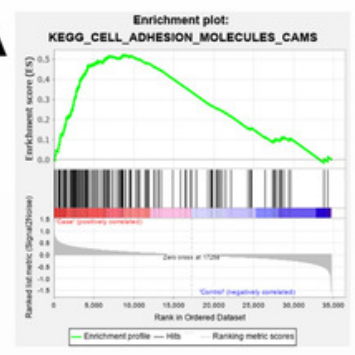

B

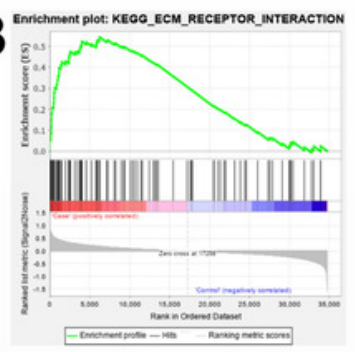

E
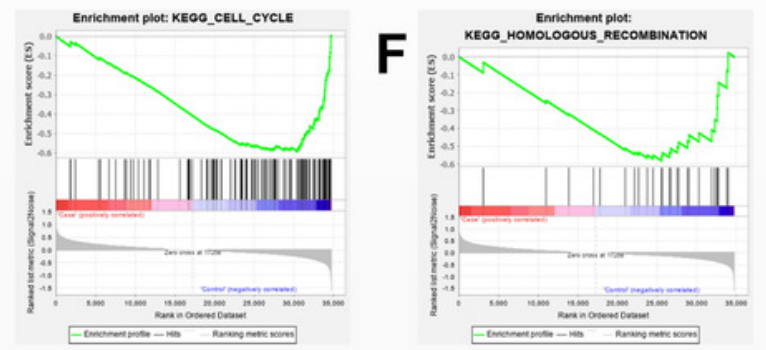

G

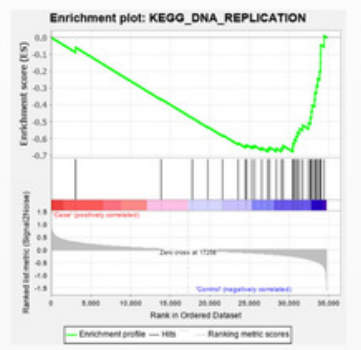

D

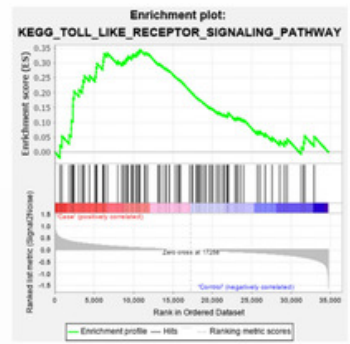

H

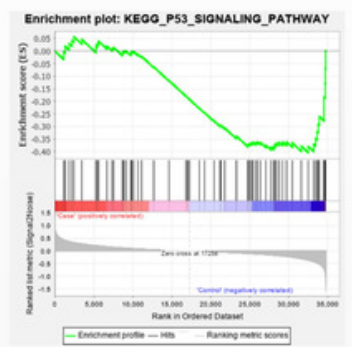


Figure 4

Construction of PPI networks and analysis of hub genes.

(A) The PPI network was constructed using Cytoscape. (B) The top 10 hub genes were screened using the MNC algorithm. The color of the hub gene holds importance according to the MNC algorithm.

A

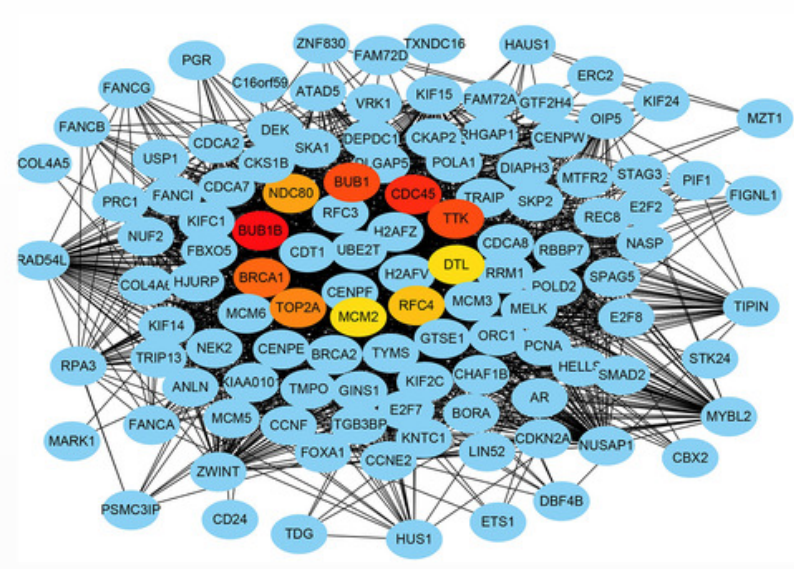

B

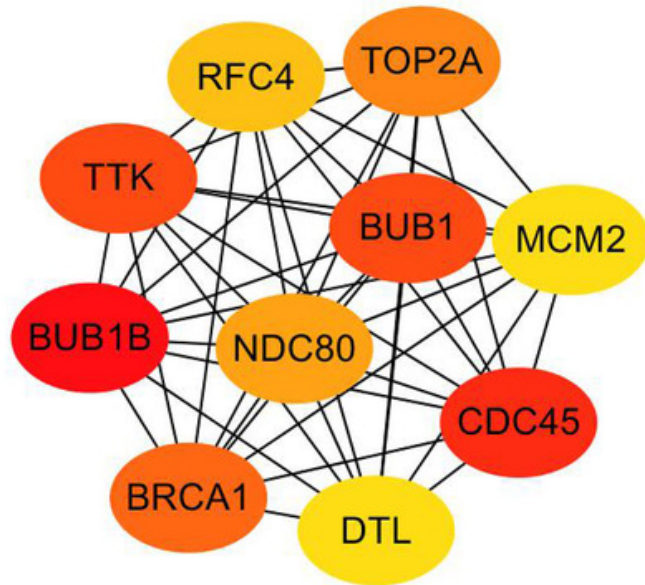


Figure 5

Analysis of hub gene expression in lung adenocarcinoma.

The red and gray boxes represent lung adenocarcinoma and adjacent normal tissue, respectively. (A) BUB1B; (B) CDC45; (C) BUB1, (D) TTK; (E) BRCA1; (F) TOP2A;(G) NDC80; (H) RFC4; (I) MCM2; and (J) DTL.
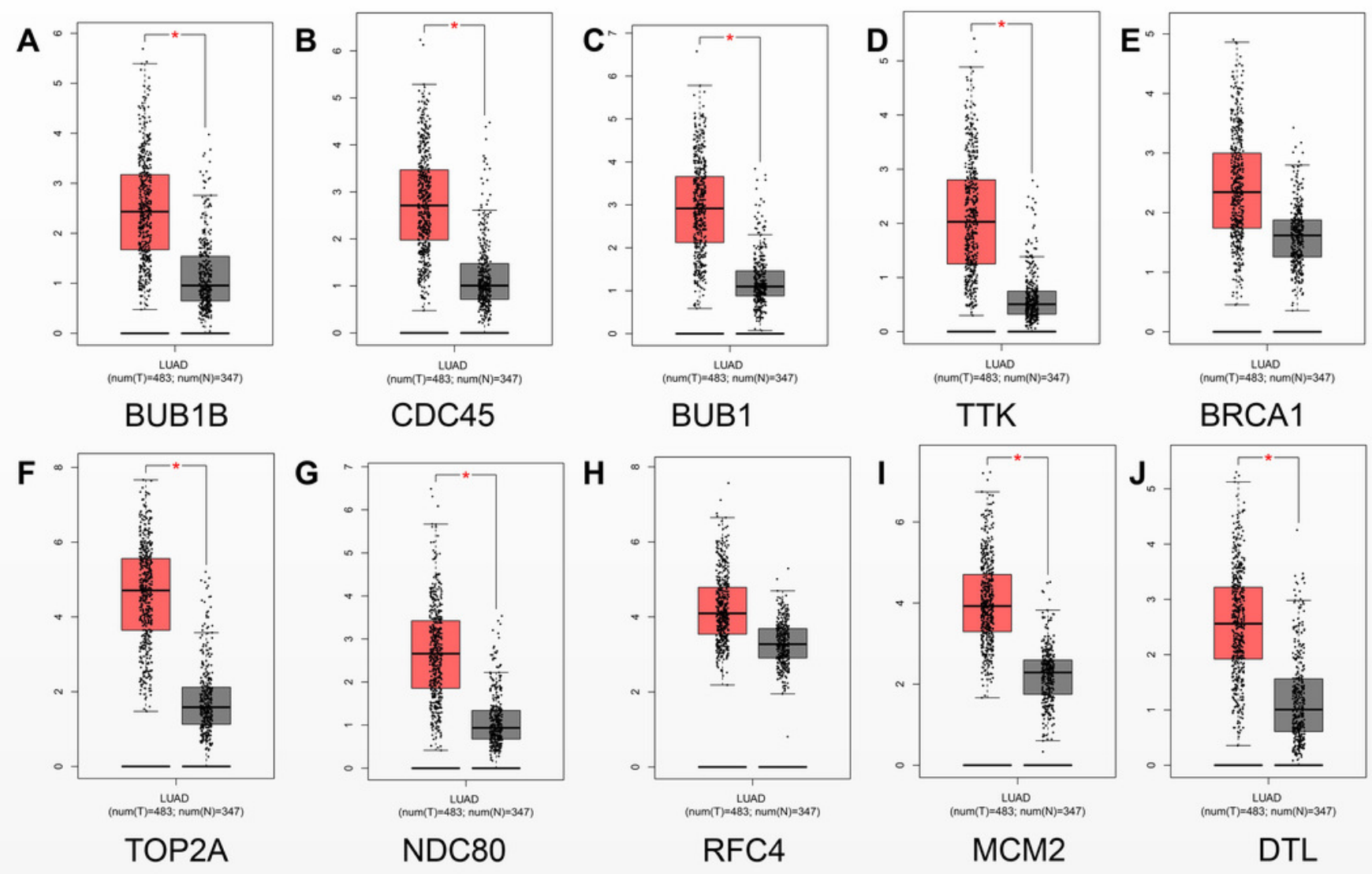
Figure 6

Analysis of the relationship between hub genes and pathological staging in lung adenocarcinoma.

(A) BUB1B; (B) CDC45; (C) BUB1; (D) TTK; (E) BRCA1; (F) TOP2A; (G) NDC80; (H) RFC4; (I) MCM2; and (J) DTL.

A.

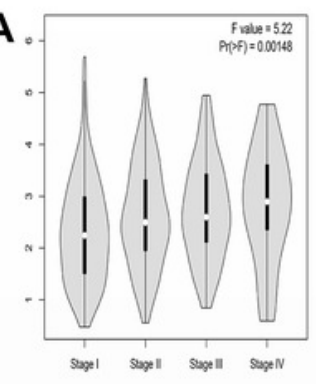

BUB1B

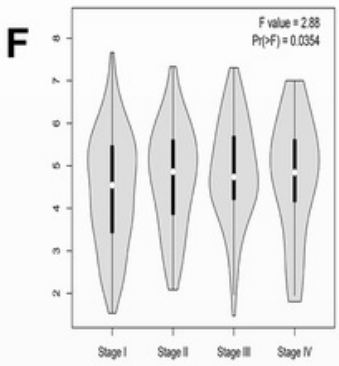

TOP2A

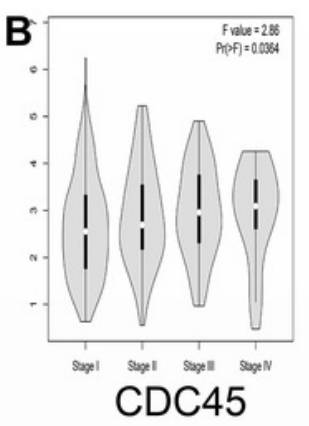

C
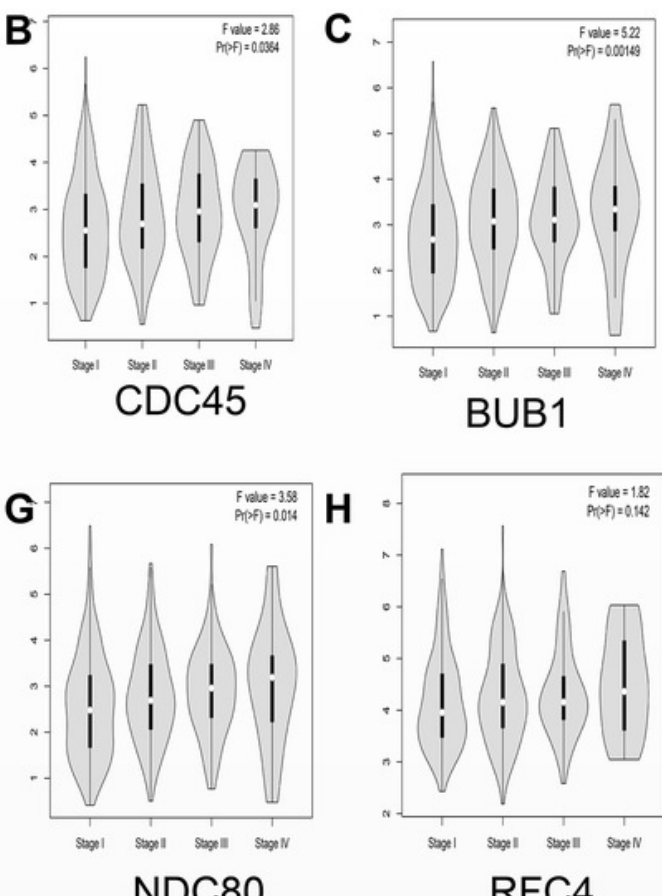

NDC80
RFC4
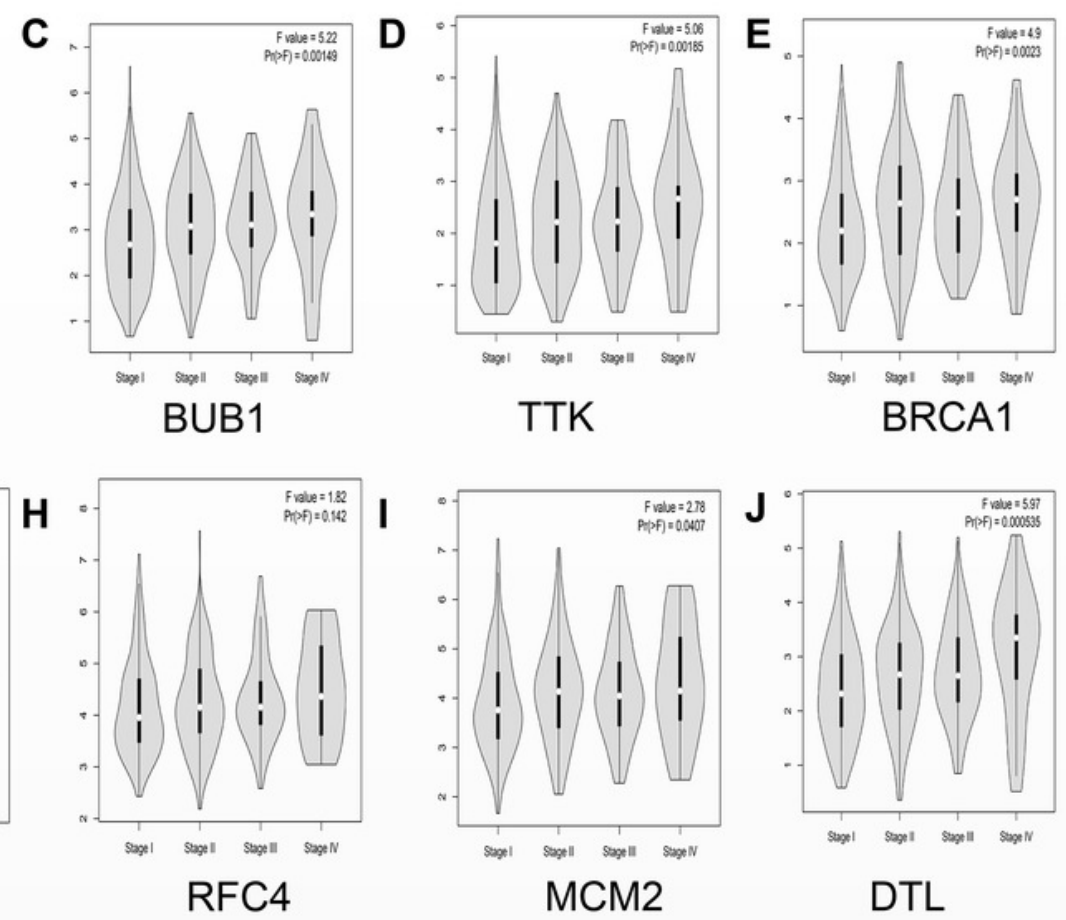
Figure 7

Survival analysis for patients with lung adenocarcinoma in relation to the expression of hub genes.

(A) BUB1B; (B) CDC45; (C) BUB1; (D) TTK; (E) BRCA1; (F) TOP2A; (G) NDC80; (H) RFC4; (I) MCM2; and (J) DTL. Red and blue represent high and low expression levels of hub genes, respectively.

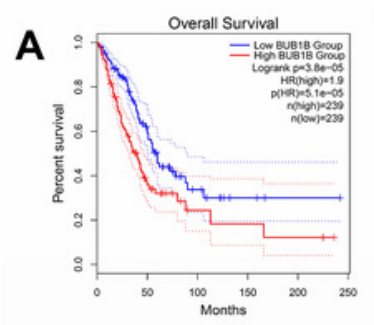

BUB1B

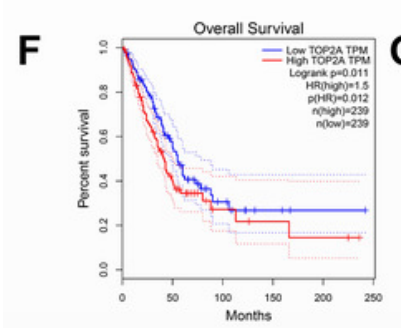

TOP2A

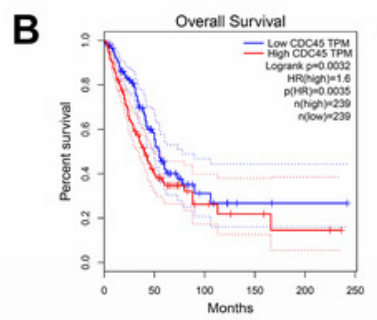

CDC45

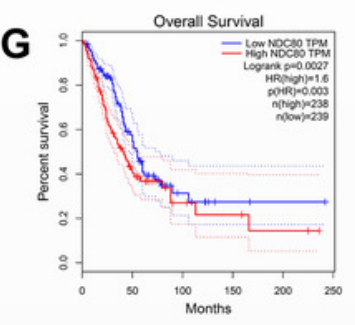

NDC80

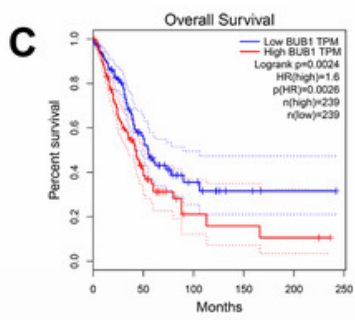

BUB1

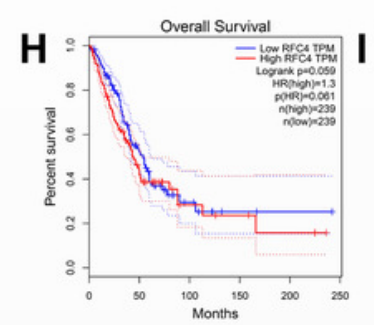

RFC4

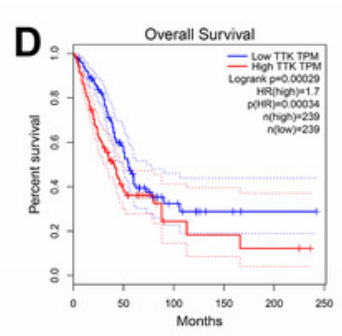

TTK

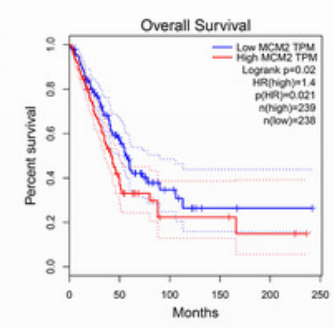

MCM2

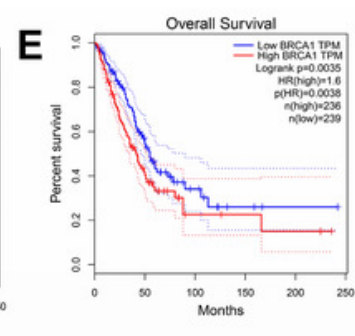

BRCA1

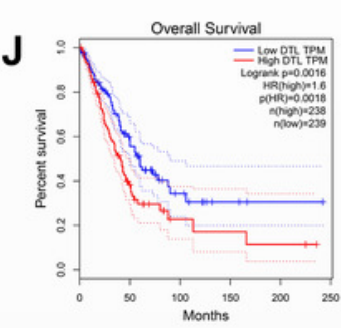

DTL 
Figure 8

Analysis of mutation characteristics of hub genes.

(A) Matrix heatmap shows genomic alterations of hub genes in five lung data sets (Broad, Cell [2012]; MSKCC, Science [2015]; TCGA, Firehose Legacy; TCGA, Nature [2014]; and TCGA, PanCancer Atlas). (B) The alteration frequencies of hub genes across five studies on lung adenocarcinoma. 
$\mathbf{A}$

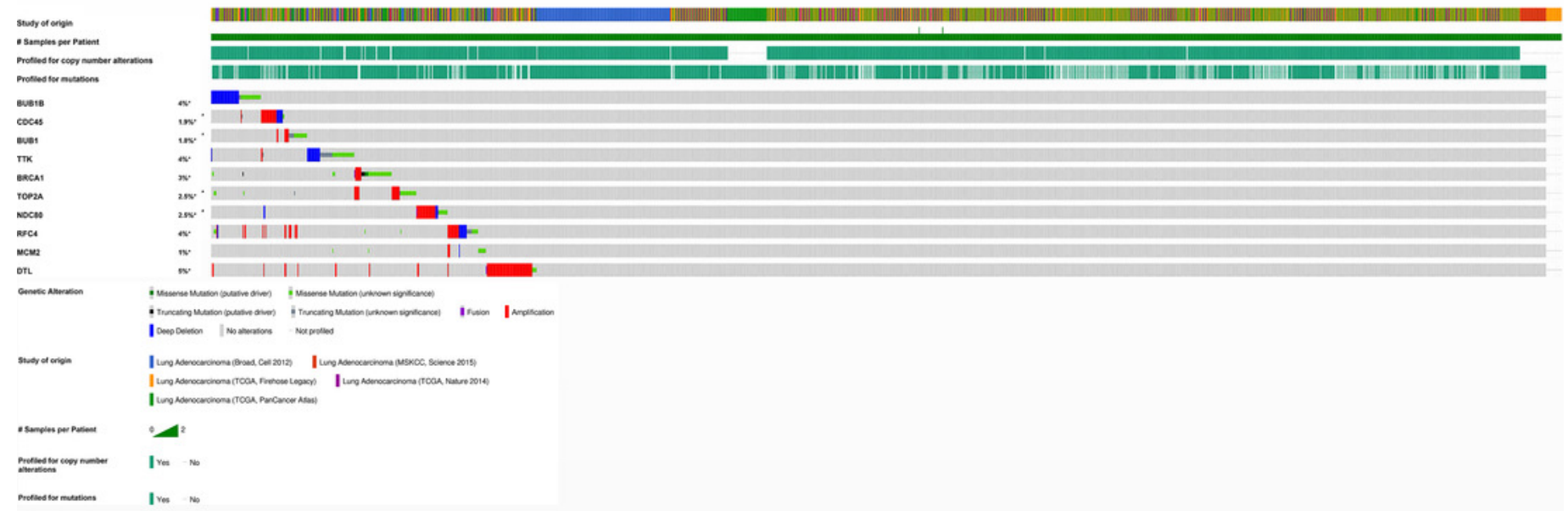

B

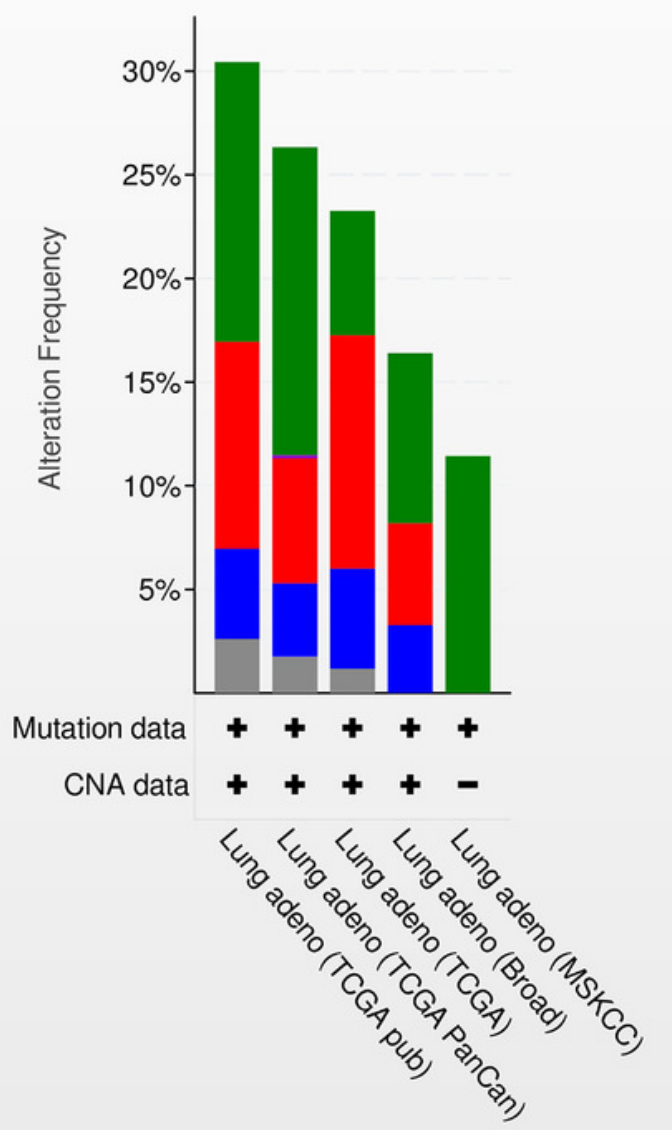

- Mutation

- Fusion

Amplification

- Deep Deletion

- Multiple Alterations 
Figure 9

Analysis of pathways of Hub genes.

(A) Pie chart of hub genes involved in pathways. (B) Interaction map of hub genes and pathways in lung adenocarcinoma.

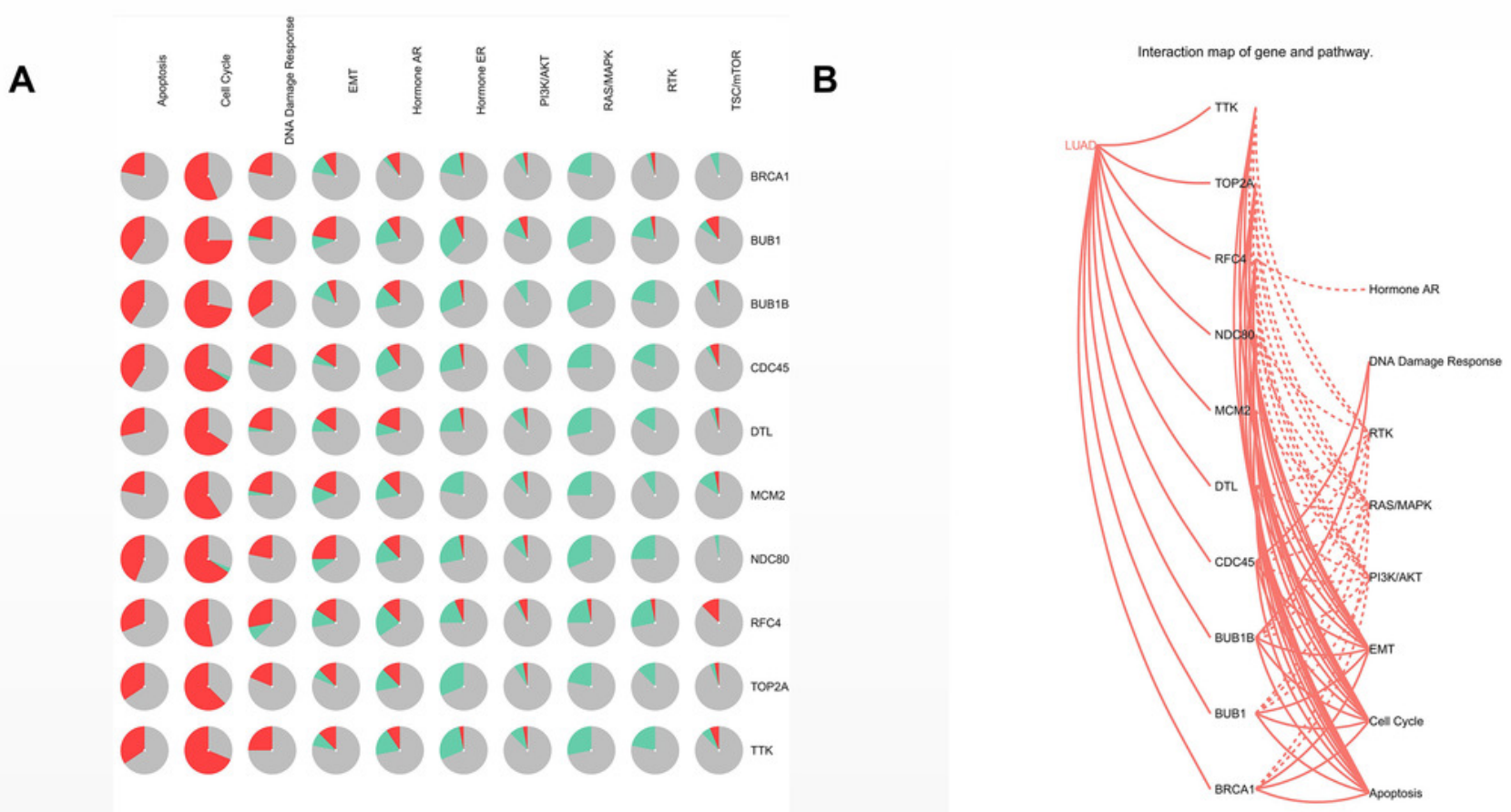


Table $\mathbf{1}$ (on next page)

Top 10 GO Terms 
1 Table 1 Top 10 GO Terms

\begin{tabular}{|c|c|c|c|c|c|c|}
\hline \multicolumn{7}{|l|}{ PDL1 positive group } \\
\hline Category & Term & Count & \multicolumn{2}{|c|}{$\%$} & \multicolumn{2}{|l|}{ P-Value } \\
\hline GOTERM_BP_FAT & cell adhesion & & 21 & 8.713692946 & $2.88 \mathrm{E}-04$ & 0.469256078 \\
\hline GOTERM_BP_FAT & biological adhesion & & 21 & 8.713692946 & $2.94 \mathrm{E}-04$ & 0.478025436 \\
\hline GOTERM_BP_FAT & cell-cell adhesion & & 12 & 4.979253112 & 5.17E-04 & 0.841085346 \\
\hline GOTERM_BP_FAT & regulation of cell motion & & 10 & 4.149377593 & $5.50 \mathrm{E}-04$ & 0.894179746 \\
\hline GOTERM_BP_FAT & MAPKKK cascade & & 9 & 3.734439834 & 0.00173479 & 2.79437728 \\
\hline GOTERM_CC_FAT & plasma membrane & & 81 & 33.60995851 & $1.28 \mathrm{E}-08$ & $1.64 \mathrm{E}-05$ \\
\hline GOTERM_CC_FAT & intrinsic to plasma membrane & & 33 & 13.69294606 & $2.53 \mathrm{E}-05$ & 0.032468756 \\
\hline GOTERM_CC_FAT & intrinsic to membrane & & 93 & 38.58921162 & $5.00 \mathrm{E}-05$ & 0.064061563 \\
\hline GOTERM_CC_FAT & integral to plasma membrane & & 31 & 12.86307054 & $1.03 \mathrm{E}-04$ & 0.132137329 \\
\hline GOTERM_CC_FAT & membrane raft & & 8 & 3.319502075 & 0.001913071 & 2.423429637 \\
\hline GOTERM_MF_FAT & cadmium ion binding & & 3 & 1.244813278 & 0.006756465 & 8.971494974 \\
\hline GOTERM_MF_FAT & sugar binding & & 8 & 3.319502075 & 0.012297355 & 15.76520126 \\
\hline GOTERM_MF_FAT & carbohydrate binding & & 11 & 4.564315353 & 0.01503554 & 18.94591653 \\
\hline GOTERM_MF_FAT & calcium ion binding & & 20 & 8.298755187 & 0.024264811 & 28.86459093 \\
\hline GOTERM_MF_FAT & lipid binding & & 12 & 4.979253112 & 0.02867722 & 33.1973471 \\
\hline \multicolumn{7}{|l|}{ PDL1 negative group } \\
\hline Category & Term & Count & $\%$ & & P-Value & $\mathrm{R}$ \\
\hline GOTERM_BP_DIRECT & cell division & & 34 & 8.629441624 & $4.29 \mathrm{E}-15$ & $7.22 \mathrm{E}-12$ \\
\hline GOTERM_BP_DIRECT & DNA replication & & 22 & 5.583756345 & $4.68 \mathrm{E}-13$ & $7.79 \mathrm{E}-10$ \\
\hline
\end{tabular}




\begin{tabular}{|c|c|c|c|c|c|}
\hline GOTERM_BP_DIRECT & mitotic nuclear division & 26 & 6.598984772 & $2.39 \mathrm{E}-12$ & $3.98 \mathrm{E}-09$ \\
\hline GOTERM_BP_DIRECT & chromosome segregation & 14 & 3.553299492 & $1.75 \mathrm{E}-10$ & 0.000000291 \\
\hline GOTERM_BP_DIRECT & $\begin{array}{l}\text { G1/S transition of mitotic cell } \\
\text { cycle }\end{array}$ & 15 & 3.807106599 & $3.32 \mathrm{E}-09$ & 0.00000554 \\
\hline GOTERM_CC_DIRECT & nucleoplasm & 89 & 22.58883249 & $1.26 \mathrm{E}-09$ & 0.00000169 \\
\hline GOTERM_CC_DIRECT & $\begin{array}{l}\text { condensed chromosome } \\
\text { kinetochore }\end{array}$ & 14 & 3.553299492 & $2.29 \mathrm{E}-09$ & 0.00000306 \\
\hline GOTERM_CC_DIRECT & nucleus & 136 & 34.5177665 & $6.77 \mathrm{E}-08$ & 0.0000906 \\
\hline GOTERM_CC_DIRECT & chromosome, centromeric region & 10 & 2.538071066 & 0.00000043 & 0.000576 \\
\hline GOTERM_CC_DIRECT & kinetochore & 11 & 2.791878173 & 0.00000104 & 0.001390211 \\
\hline GOTERM_MF_DIRECT & DNA binding & 52 & 13.19796954 & 0.000106 & 0.150583673 \\
\hline GOTERM_MF_DIRECT & protein binding & 190 & 48.22335025 & 0.000124 & 0.175586276 \\
\hline GOTERM_MF_DIRECT & chromatin binding & 19 & 4.822335025 & 0.000252 & 0.356042613 \\
\hline GOTERM_MF_DIRECT & DNA helicase activity & 5 & 1.269035533 & 0.000804 & 1.132060211 \\
\hline GOTERM_MF_DIRECT & ATP binding & 43 & 10.91370558 & 0.002267608 & 3.161395589 \\
\hline
\end{tabular}


Table 2 (on next page)

Top 10 KEGG pathway enrichment results 
1 Table 2 Top 10 KEGG pathway enrichment results

\begin{tabular}{|c|c|c|c|c|c|}
\hline \multicolumn{6}{|l|}{ PDL1 positive group } \\
\hline Category & Term & Count \% & P-Value & Genes & FDR \\
\hline KEGG_PATHWAY & $\begin{array}{l}\text { Arrhythmogenic right } \\
\text { ventricular cardiomyopathy } \\
\text { (ARVC) }\end{array}$ & 72.904564315 & 0.001300189 & $\begin{array}{l}\text { LAMA2, ITGA9, } \\
\text { CACNA2D1, RYR2, } \\
\text { ITGA10, CACNA2D3, } \\
\text { CTNNA3 }\end{array}$ & 1.414211029 \\
\hline KEGG_PATHWAY & $\begin{array}{l}\text { Cell adhesion molecules } \\
\text { (CAMs) }\end{array}$ & 72.904564315 & 0.019094239 & $\begin{array}{l}\text { NCAM2, ITGA9, SELP, } \\
\text { CDH15, CD22, CLDN22, } \\
\text { HLA-DQA1 }\end{array}$ & 19.02707303 \\
\hline KEGG_PATHWAY & PPAR signaling pathway & 52.074688797 & 0.024587412 & $\begin{array}{l}\text { LPL, SLC27A1, OLR1, } \\
\text { FABP3, ANGPTL4 }\end{array}$ & 23.85529626 \\
\hline KEGG_PATHWAY & $\begin{array}{l}\text { Complement and coagulation } \\
\text { cascades }\end{array}$ & 52.074688797 & 0.024587412 & $\begin{array}{l}\text { KNG1, CD55, CR2, F3, } \\
\text { CFD }\end{array}$ & 23.85529626 \\
\hline KEGG_PATHWAY & ECM-receptor interaction & 52.074688797 & 0.045850772 & $\begin{array}{l}\text { LAMA2, ITGA9, ITGA10, } \\
\text { CHAD, THBS4 }\end{array}$ & 40.17953232 \\
\hline \multicolumn{6}{|l|}{ PDL1 negative group } \\
\hline KEGG_PATHWAY & DNA replication & 102.538071066 & 4.44E-09 & $\begin{array}{l}\text { RFC3, RFC4, POLD2, } \\
\text { PCNA, POLA1, MCM2, } \\
\text { MCM3, MCM5, CM6, } \\
\text { RPA3 }\end{array}$ & 0.00000536 \\
\hline KEGG_PATHWAY & Cell cycle & 153.807106599 & $1.25 \mathrm{E}-08$ & $\begin{array}{l}\text { E2F2, SKP2, TTK, } \\
\text { SMAD2, MCM2, } \\
\text { MCM3, MCM5, MCM6, } \\
\text { CCNE2, CDC45, } \\
\text { CDKN2A, PCNA, BUB1, } \\
\text { BUB1B, ORC1 }\end{array}$ & 0.0000151 \\
\hline KEGG_PATHWAY & Mismatch repair & 51.269035533 & 0.000497 & RFC3, RFC4, POLD2, & 0.598123244 \\
\hline
\end{tabular}


PCNA, RPA3

KEGG_PATHWAY Nucleotide excision repair

KEGG_PATHWAY p53 signaling pathway

\author{
RFC3, RFC4, POLD2, \\ $\begin{array}{lll}6 & 1.52284264 & 0.001008028\end{array}$ \\ PCNA, GTF2H4, RPA3 \\ 1.210410161 \\ CCNE2, CDKN2A, \\ $6 \quad 1.522842640 .004883612$ SERPINB5, RPRM, PERP, 5.740021178 \\ GTSE1
}

2 
Table 3 (on next page)

Top 10 hub genes ranked using the MNC method 
1 Table 3 Top 10 hub genes ranked using the MNC method

\begin{tabular}{|c|c|c|}
\hline Rank & Name & Score \\
\hline 1 & BUB1B & 81 \\
\hline 2 & $\mathrm{CDC} 45$ & 79 \\
\hline 3 & BUB1 & 78 \\
\hline 3 & TTK & 78 \\
\hline 5 & BRCA1 & 75 \\
\hline 6 & TOP2A & 73 \\
\hline 7 & NDC80 & 72 \\
\hline 8 & RFC4 & 71 \\
\hline 9 & MCM2 & 69 \\
\hline 9 & DTL & 69 \\
\hline
\end{tabular}

